\title{
The Existence of a Putative Regulatory Element in 3'-Untranslated Region of Proto-oncogene HOX11's mRNA
}

\author{
Yue Li ${ }^{\dagger,}$, Zhao-Zhao Jiang ${ }^{\dagger,}$, Hai-Xu Chen \\ Health Science Center, Shanghai Institutes for Biological Sciences, Chinese Academy of Sciences and \\ Shanghai Second Medical University, Shanghai, China \\ "Shanghai Research Center of Biotechnology, Shanghai Institutes for Biological Sciences, Chinese Academy of Sciences, Shanghai, China \\ ${ }^{\S}$ Department of Medicine and Therapeutics, Prince of Wales Hospital, The Chinese University of Hong Kong, Shatin, Hong Kong
}

\section{Received 7 November 2004, Accepted 7 February 2005}

\begin{abstract}
HOX11 encodes a homeodomain-containing transcription factor which directs the development of the spleen during embryogenesis. While HOX11 expression is normally silenced through an unknown mechanism in all tissues by adulthood, the deregulation of $\mathrm{HOX} 11$ expression is associated with leukemia, such as T-cell acute lymphoblastic leukemia. The elucidation of regulatory elements contributing to the molecular mechanism underlying the regulation of HOX11 gene expression is of great importance. Previous reports of $\mathrm{HOX} 11$ regulatory elements mainly focused on the 5'-flanking region of $\mathrm{HOX} 11$ on the chromosome related to transcriptional control. To expand the search of putative cis-elements involved in $H O X 11$ regulation at the post-transcriptional level, we analyzed $H O X 11$ mRNA 3'untranslated region (3'UTR) and found an AU-rich region. To characterize this AU-rich region, in vitro analysis of HOX11 mRNA 3'UTR was performed with human RNAbinding protein HuR, which interacts with AU-rich element (ARE) existing in the 3'UTR of many growth factors' and cytokines' mRNAs. Our results showed that the HOX11 mRNA 3'UTR can specifically bind with human HuR protein in vitro. This specific binding could be competed effectively by typical ARE containing RNA. After the deletion of the AU-rich region present in the HOX11 mRNA 3'UTR, the interaction of HOX11 mRNA 3'UTR with HuR protein was abolished. These findings suggest that HOX11 mRNA 3'UTR contains cis-acting element which shares similarity in the action pattern with ARE-HuR interactions and may involve in the posttranscriptional regulation of the $\mathrm{HOX} 11$ gene.
\end{abstract}

Keywords: AU-rich element, $H O X 11$, Proto-oncogene, T-cell acute lymphoblastic leukemia, 3'-untranslated region

\footnotetext{
*To whom correspondence should be addressed.

Tel: 86-21-63852596; Fax: 86-21-63852655

E-mail:wjma@sibs.ac.cn
}

\section{Introduction}

HOX11 encodes a homeodomain-containing transcription factor which binds to a specific DNA sequence and is localized in the nucleus (Dear et al., 1993; Zhang, N. et al., 1993). It can transcriptionally transactivate a variety of promoters in both yeast and mammalian cells (Zhang, N. et al., 1996). HOX11 homologues exist in mice (Dear et al., 1993), Drosophila (Dear and Rabbitts, 1994), and Xenopus (Patterson and Krieg, 1999).The normal function of the $H O X 11$ protein is to direct the development of the spleen during embryogenesis. While HOX11 expression is normally silenced through an unknown mechanism in all tissues by adulthood (Roberts et al., 1994; Dear et al., 1995; Kanzler and Dear, 2001). Deregulation of HOXI1 is associated with leukemia, such as human T-cell acute lymphoblastic leukemia (T-ALL). Aberrant expression of HOXI1 could be activated by chromosomal translocations $\mathrm{t}(7 ; 10)$ and $\mathrm{t}(10 ; 14)$, which occurs in 5-10 \% of T-ALL (Kennedy et al., 1991; Lichty et al., 1995). Recent research suggests the existence of alternative mechanisms of $\mathrm{HOX} 11$ deregulation that accounts for a much large proportion of T-ALL patient specimens with HOX11 expression in the absence of chromosome translocation (Kees et al., 2003).

Because of the important role of HOX11 in the development of spleen and its deregulation closely associated with leukemia, the elucidation of regulatory elements contributing to the molecular mechanism underlying the regulation of $H O X 11$ gene expression is of great interest. The research of $H O X 11$ regulatory elements previously reported mainly focused on the 5'-flanking region on the chromosome related to transcriptional control. Both positive and negative transcriptional regulatory cis-elements of $\mathrm{HOX} 11$ were found at the 5'-flanking region of $H O X 11$ gene (Brake et al., 1998; Brake et al., 2002). HOXI1 expression requires protein expression and may act as a delayed early response gene (Zhang, N. et al., 1995). Many early response genes' mRNAs 
are targeted for post-transcriptional regulation by virtue of ciselements in the 3'-untranslated region (3'UTR) (Shaw and Kamen, 1986; Sachs, 1993; Levy et al., 1996). So is there any cis-element in the 3'UTR of HOX11 mRNA? A scan of putative cis-element in HOX11 mRNA sequence was performed, and we found a segment of AU-rich sequence in 3'UTR of HOX11 mRNA, which is similar to the AU-rich element (ARE). We anticipate this AU-rich segment in 3'UTR of HOX11 may be a cis-element. In order to characterize this element, RNA-protein interaction analysis is performed using human RNA binding protein HuR, which can bind specifically with typical cis-element ARE (Ma et al., 1996). In vivo studies have showed HuR can specifically bind and stabilize ARE-containing mRNA (Fan and Steitz, 1998; Peng et al., 1998; Levy et al., 1998). Over-expression of HuR is found in tumors of central nervous system, and strong HuR expression was limited to high grade malignancies (Nabors et al., 2001). With RNA-protein interaction analysis, we can evaluate the binding affinity and specificity of $H O X 11$ mRNA 3'UTR with HuR protein, compare the binding features in the competition assay with typical ARE containing RNA, and determine the main binding site by deletion analysis.

\section{Materials and Methods}

Cloning and construction of human HOX11 mRNA 3'UTR in vitro transcription plasmids The human HOX11 mRNA 3'UTR was cloned by PCR as follows. The genomic DNA was extracted from human blood cells by using QIAamp DNA Blood mini kit and used as template to amplify the 3'UTR of human HOX11 mRNA encoded by the third exon of HOX11 gene (GenBank accession no. AJ009794). The following oligodeoxynucleotides were used in the amplification of the human HOX11 mRNA 3'UTR: forward, 5'-TG AGCCTGCCCATTCTG-3'; reverse, 5'-TTCACATAAATTACAC AAGCAC-3'. The PCR products were subcloned into a pUCm-T vector (Shenergy Biocolor Biological Science \& Technology Company, Shanghai, China). The resultant plasmid was called pUCm-HOX11. The HOX11 mRNA 3'UTR which lacks the AUrich sequence was amplified using oligodeoxynucleotides described as follows: forward, 5'-GATTCAGAGAAAGGCAAGGGAGGT-3'; reverse, 5'-CAGTTCAGACAGGTGCGGGAC-3'. The pUCm-HOX11 plasmid acted as template, and the PCR products was self linked. The resultant plasmid was called pUCm-HOX11 $\Delta$. Both the cloned pUCm-HOX11 and pUCm-HOX11 $\Delta$ were verified by sequencing.

Preparation of RNA transcripts Plasmid DNAs were digested with the appropriate restriction enzymes and transcribed using MAXIscript in vitro transcription kit (Ambion). For the synthesis of biotin-labeled RNA, 50\% CTP were replaced by biotin-14-CTP (Invitrogen, San Diego, USA); for the synthesis of unlabeled RNA, no biotin-14-CTP was added. pUCm-HOX11 was linearized with Sal I and transcribed with T7 RNA polymerase, yielding a 938 nucleotides transcript (called HOX11-3'UTR) containing the sequence from residues 1003 to 1850 of HOX11 mRNA (GenBank accession no. M75952), which located in the downstream of the HOX11 mRNA stop codon. pUCm-HOX11 1 was linearized with
Sal I and transcribed with T7 RNA polymerase, yielding an 888 nucleotides transcript (called HOX11-3'UTR $\Delta$ ) containing the sequence from residues 1003 to 1850 of HOX11 mRNA (GenBank accession no. M75952), which located in downstream of the HOX11 mRNA stop codon while lacking the AU-rich sequence from residues 1241 to 1290 of HOX11 mRNA. pAUFL was linearized with Hind III, yielding a transcript of 214 nucleotides containing the sequence from residues 568 to 781 downstream of the $c$-fos mRNA stop codon (Shyu et al., 1991). pSP65H $\gamma$ (human $\gamma$-globin) was linearized with Sau3A I, yielding 165 nucleotides, containing 80 nucleotides of coding sequence and 85 nucleotides of 3'-untranslated regions (Chung et al., 1996). The biotin-labeled RNA concentration was determined by measuring $\mathrm{OD}_{260}$ with Gene Spec III (Naka instruments Co. Ltd.)

Purification of GST-HuR fusion protein The GST (glutathione $S$-transferase) -HuR fusion protein was expressed from pGEX-HuR (Ma et al., 1996). An overnight culture of E. coli BL 21, transformed with pGEX-HuR, was diluted in $50 \mathrm{ml} \mathrm{LB}$ medium at a proportion of $0.5: 50$ and incubated at $37^{\circ} \mathrm{C}$ for $2 \mathrm{~h}$. The culture was induced with IPTG $(0.04 \mathrm{mM})$. After $4 \mathrm{~h}$ of further growth at $30^{\circ} \mathrm{C} \mathrm{E}$. coli cells were spun down and resuspended in $5 \mathrm{ml}$ of buffer A (50 mM Tris pH 8.0, $200 \mathrm{mM} \mathrm{NaCl}, 1 \mathrm{mM}$ ethylenediamine tetraacetic acid (EDTA)). The cells were lysed by adding lysozyme to a final concentration of $0.2 \mathrm{mg} / \mathrm{ml}$ and Triton X-100 to $1 \%$, respectively. The lysate was centrifuged at $12,000 \times g$ for $30 \mathrm{~min}$. The resultant supernatant was mixed with $300 \mu$ of $50 \%$ slurry of Glutathione Sepharose 4B (Pharmacia Biotech, Uppsala, Sweden) and incubated with gentle agitation at room temperature for $1 \mathrm{~h}$. Mixtures were centrifuged at $1000 \times g$ for $5 \mathrm{~min}$. After washing the pellet three times with $1.5 \mathrm{ml}$ buffer $\mathrm{A}$, the bound protein was eluted with elution buffer $(50 \mathrm{mM}$ Tris $\mathrm{pH} 8.0,10 \mathrm{mM}$ reduced glutathione). The GST-HuR fusion protein concentration was measured using Bradford assay (Bradford, 1976).

Electrophoretic mobility shift assay Excess amounts of tRNA and bovine serum albumin (BSA) were used as non-specific competitor to assure the specificity of mRNA-protein interaction in electrophoretic mobility shift assay (EMSA). Reaction mixtures (0.02 ml) contained $50 \mathrm{mM}$ Tris ( $\mathrm{pH} 7.0), 150 \mathrm{mM} \mathrm{NaCl}, 0.25 \mathrm{mg} /$ $\mathrm{ml}$ tRNA, $0.25 \mathrm{mg} / \mathrm{ml}$ BSA, 40 fmol biotin-labeled RNA transcripts or $200 \mathrm{fmol}$ biotin-labeled $\gamma$-globin and protein as indicated. Mixtures were incubated at $37^{\circ} \mathrm{C}$ for $10 \mathrm{~min}$. Following incubation, $2 \mu \mathrm{l}$ of a dye mixture (50\% glycerol, $0.025 \%$ bromphenol blue, $0.025 \%$ xylene cyanol) was added and the reaction mixture was immediately loaded on a $1 \%$ agarose gel in TBE buffer $(40 \mathrm{mM}$ Tris borate, $1 \mathrm{mM}$ EDTA, $\mathrm{pH}$ 8.0). The gel was then electrophoresed at $70 \mathrm{~V}$ for $45 \mathrm{~min}$.

Detection of the biotin-labeled RNA and quantification of its optical density The RNA was transferred from gel to Zeta-probe nylon membrane (BIO-RAD Co., Richmond, USA) using standard capillary transferring (Sambrook et al., 1992). The RNA was crosslinked to membrane by exposure to $302 \mathrm{~nm}$ ultraviolet radiation on a UV transilluminator for $3 \mathrm{~min}$. The membrane was incubated in $10 \mathrm{ml}$ block solution (0.1 M Tris, PH 7.5, $0.1 \mathrm{M} \mathrm{NaCl}, 2 \mathrm{mM}$ $\mathrm{MgCl}_{2}, 3 \%$ bovine serum albumin Fraction $\mathrm{V}$ ) at $30^{\circ} \mathrm{C}$ for $1 \mathrm{~h}$ and then incubated at $30^{\circ} \mathrm{C}$ with $0.85 \mu \mathrm{g} / \mathrm{ml}$ Streptavidin Alkaline 
Phosphatase (Promega, Madison, USA) in $10 \mathrm{ml}$ AP 7.5 buffer (0.1 M Tris, PH 7.5, $0.1 \mathrm{M} \mathrm{NaCl}, 2 \mathrm{mM} \mathrm{MgCl}$ ) for $10 \mathrm{~min}$. After being washed twice with $100 \mathrm{ml}$ AP 7.5 buffer for $10 \mathrm{~min}$ and once with $100 \mathrm{ml}$ AP 9.5 buffer $(0.1 \mathrm{M}$ Tris, PH $9.5,0.1 \mathrm{M} \mathrm{NaCl}$, $50 \mathrm{mM} \mathrm{MgCl}_{2}$ ) for $10 \mathrm{~min}$, the membrane was incubated with $2.5 \mathrm{mg}$ nitroblue tetrazolium (NBT) and $1.25 \mathrm{mg}$ 5-bromo-4-chloro-3indolyl phosphate (BCIP) in $7.5 \mathrm{ml} \mathrm{AP} 9.5$ buffer at room temperature for $15 \mathrm{~min}$, and the TE buffer $(10 \mathrm{mM}$ Tris PH 8.0 , $1 \mathrm{mM}$ EDTA) was added to stop the reaction.

After the color development reaction of biotin-labeled RNA, the nylon membrane was scanned by a scanner. The optical density of each band was quantified using ImageQuant version 5.2 software (Molecular Dynamics). The integrated density of all the pixels in the area of each band was quantified and adjusted by a subtraction of density in nearest blank area of the same size (background). This value is the optical density of the band. A series quantities of biotinlabeled HOX11-3'UTR samples were processed and the optical densities of the corresponding band were measured. In the range of the processed RNA quantities, the optical density of biotin-labeled HOX11-3'UTR versus the logarithm value of RNA quantities reveals a straight line. With this standard curve, the biotin-labeled HOX11-3'UTR quantities could be calculated according to the optical density of biotin-labeled HOX11-3'UTR on the membrane within the linear range and the results of EMSA were quantitatively analyzed (Li et al., 2004).

The apparent equilibrium dissociation constant $\mathrm{K}_{\mathrm{d}}$, which represents the binding affinity between two molecules, is calculated based on a plotting of $\mathrm{Lg}\{[\mathrm{RNA}-\mathrm{Protein}] /[\mathrm{RNA}]\}$ on the Y-axis and $\mathrm{Lg}$ [protein] on the X-axis (Chung et al., 1996).

\section{Results}

The HOX11-3'UTR can be bound with HuR protein in a concentration-dependent pattern In electrophoretic mobility shift assay (EMSA), purified GST-HuR fusion protein was added to the RNA-binding buffer at indicated amounts immediately prior to the binding reaction. As shown in Fig. 1, purified GST-HuR fusion protein converts the HOX11-3'UTR to a stable protein-RNA complex that migrates slowly on agarose gel electrophoresis (Fig. 1, lanes 3-8) than the HOX11-3'UTR alone (Fig. 1, lane 1). When the concentration of GST-HuR increased from $25 \mathrm{nM}$ to $800 \mathrm{nM}$, the amount of the formed protein-RNA complex increased, and the unbound HOX11-3'UTR reduced gradually (Fig. 1, lanes 3-8). The complex is formed between HOX11-3'UTR and HuR protein, since no complex was observed by HOX11-3'UTR and purified GST (Fig. 1, lane 2). When the concentration of GST$\mathrm{HuR}$ was at $400 \mathrm{nM}$ to $800 \mathrm{nM}$, retarded HOX11-3'UTR and HuR complex varied in size and distributed in certain range (Fig. 1, lanes 7 and 8). The result indicated that the HOX113'UTR can be bounded by human HuR protein specifically in a concentration-dependent pattern.

Quantitative analysis to the specific binding of the HOX11-3'UTR with HuR protein A series of biotin-labeled

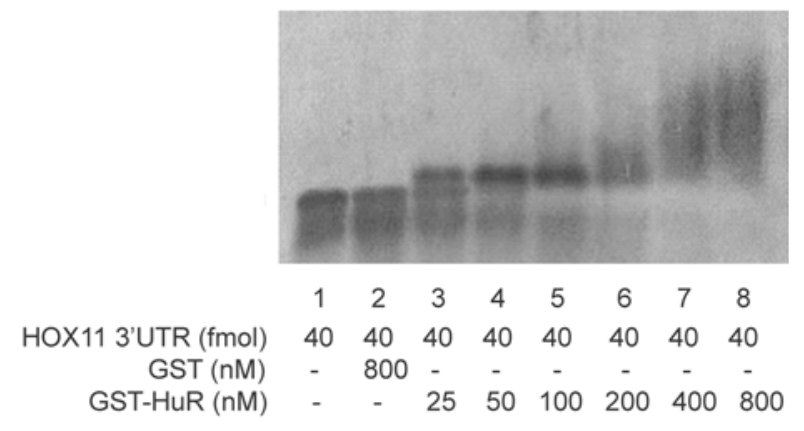

Fig. 1. EMSA of HOX11-3'UTR and HuR protein. The indicated concentrations of GST or HuR were mixed with 40 fmol biotinlabeled HOX11-3'UTR. Following incubation at $37^{\circ} \mathrm{C}$ for 10 $\mathrm{min}$, the reaction mixtures were resolved on $1 \%$ agarose gel.

HOX11-3'UTR samples whose quantities are 1.27, 3.81, 11.4, 34.3 and 103 fmol respectively were processed and their corresponding optical densities were measured after electrophoresis and being transferred to the nylon membrane. A plot of the optical density of biotin-labeled HOX11-3'UTR versus the logarithm value of HOX11-3'UTR quantities revealed a straight line within this range and served as the standard curve. With this standard curve of the relationship between the optical densities of biotin-labeled HOX11-3'UTR and the logarithm value of quantities of the corresponding RNA, the biotin-labeled RNA quantity on the membrane was determined after calculation.

This quantitative approach was employed to measure RNA quantities in lanes 3-8 of Fig. 1. The integrated density of all the pixels in the area of each unbound HOX11-3'UTR and retarded HOX11-3'UTR and HuR complex was quantified and adjusted by a subtraction of density in nearest blank area of the same size (background), respectively. Based on the value of the optical densities and the standard curve, HOX113'UTR quantities were determined and the values of complex/ free RNA were obtained. A plot of the logarithm value of complex/free RNA versus the logarithm value of $\mathrm{HuR}$ concentration reveals a straight line with an intersect on the $\mathrm{X}$ axis at $120 \mathrm{nM}$ (Fig. 2), which reveals the binding of $\mathrm{HuR}$ with HOX11-3'UTR is a simple molecular reaction with an apparent $\mathrm{K}_{\mathrm{d}}$ of $120 \mathrm{nM}$.

The binding of HOX11-3' UTR with HuR protein can be competed by typical ARE-containing RNA We next determined whether this specific interaction has similarity with the typical ARE-protein interaction. RNA transcript of full length AU-rich sequence in the $c$-fos 3'UTR (AUFL) which contains ARE of the $c$-fos mRNA, and RNA transcript of human $\gamma$-globin 3 ' sequence $(\gamma$-globin) which does not contain any ARE, were used as the competitors in the competition experiment. The competition experiment was made to check whether HOX11-3'UTR would be displaced by AUFL transcripts for binding with HuR protein. As shown in Fig. 3, GST-HuR protein did not bind to $\gamma$-globin RNA, which 


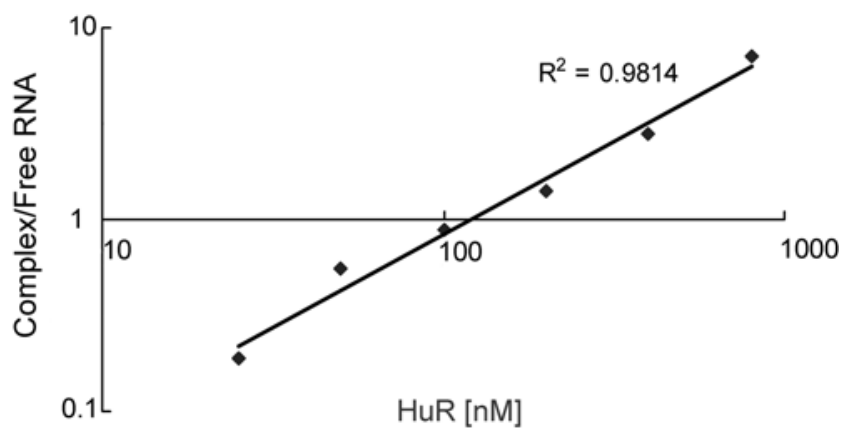

Fig. 2. Plot of $1 \mathrm{~g}$ complex/free HOX11-3'UTR versus $\lg$ HuR concentrations. Analysis of binding affinity between HOX113'UTR and HuR was performed by quantitation and analysis of EMSA results.

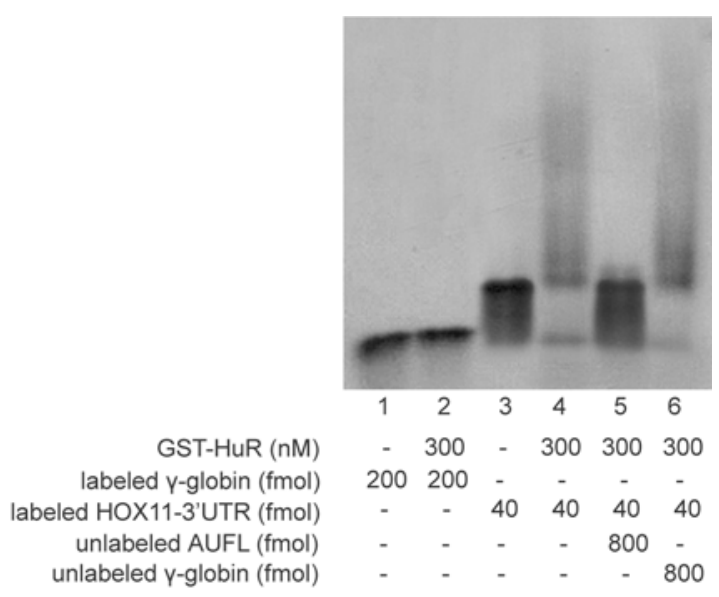

Fig. 3. Competition of binding experiment by using AUFL transcript and $\gamma$-globin RNA as the competitor. Indicated amount of biotin-labeled HOX11-3'UTR or $\gamma$-globin transcript was incubated with indicated concentration of HuR protein. Unlabeled $\gamma$-globin and AUFL transcripts acted as competitor at about 20 times excess amounts of biotin-labeled HOX11-transcripts.

was used as a negative control (Fig. 3, lanes 1 and 2). Unlabeled AUFL transcripts, acted as competitor, were added to the RNA-binding buffer at about 20 times excess amounts of biotin-labeled HOX11-3'UTR transcripts prior to the binding reaction. The binding of HuR to the HOX11-3'UTR transcripts was greatly reduced (Fig. 3, lane 5) comparing with the retarded HuR- HOX11-3'UTR complex (Fig. 3, lane $4)$. No reduced reaction was observed with unlabeled $\gamma$-globin as competitor at about 20 times excess amounts of HOX113'UTR transcripts (Fig. 3, lane 6), so complex formation was not affected comparing with the complex retardation (Fig. 3, lane 4). Thus the complex of HOX11-3'UTR and HuR protein can be inhibited by AUFL RNA. No inhibition was observed when 20-fold $\gamma$-globin mRNA were added. So it means that the AUFL RNA can effectively compete with HOX11 mRNA 3'UTR for HuR binding. The result suggests that HOXI1 mRNA 3'UTR and ARE have similarity in the binding site or action pattern with HuR protein.
(A)

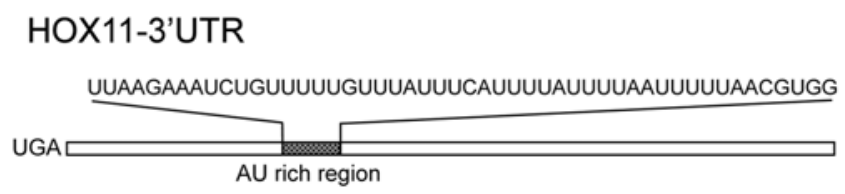

HOX11-3'UTRA

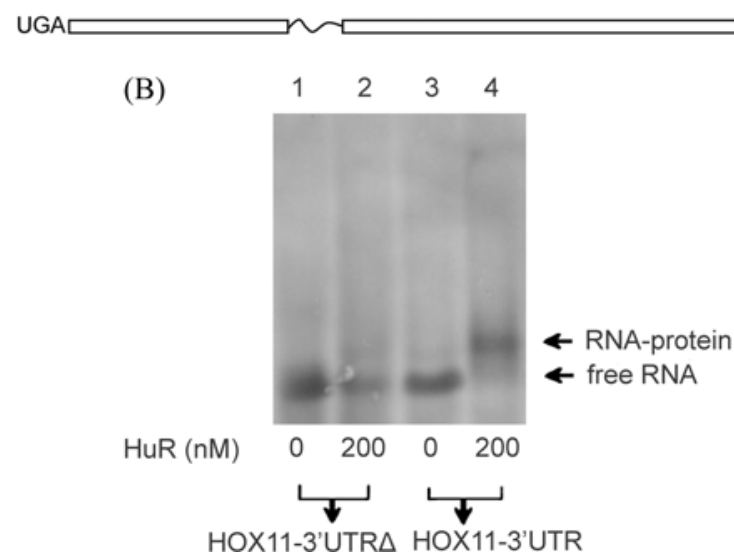

Fig. 4. Comparison of HOX11-3'UTR with HuR and HOX113'UTR $\triangle$ with HuR. A: Mapping of the HOX11-3'UTR and HOX11-3'UTRA RNA transcripts. The AU-rich sequence is showed on top of the HOX11-3'UTR, the curve represents the AU-rich sequence is deleted in HOX11-3'UTRA. B: Binding experiments of HuR protein with $40 \mathrm{fmol}$ biotin-labeled HOX113'UTR and 40 fmol biotin-labeled HOX11-3'UTR $\Delta$, respectively.

An AU-rich region in HOX11-3'UTR contributes to its specific binding with HuR Analysis of the sequence revealed that the HOX11 mRNA 3'UTR contains an AU-rich region of 50 nucleotides in the proximal portion of the 3'UTR. The location of this AU-rich region is from nt 1241 to 1290 of HOX11 mRNA (GenBank accession no. M75952). Since HOX11 mRNA 3'UTR and ARE have similarity in the binding site or action pattern with HuR protein, this AU-rich region may participate in the specific binding of HOX11 mRNA 3'UTR with HuR protein. To verify this hypothesis, a new plasmid pUCm-HOX11 $\Delta$ was constructed to produce transcript (designated HOX11-3'UTR $\Delta$ ) that contains HOX11 mRNA 3'UTR but lacks the AU-rich sequence (Fig. 4A).

We first compared the binding ability of HOX11-3'UTR and HOX11-3'UTR $\Delta$ with HuR protein. As shown in Fig. 4B, when the concentration of HuR is $200 \mathrm{nM}$, the HOX113'UTR $\Delta$ and HuR formed little complex, compared with a totally obvious complex formed by HOX11-3'UTR and HuR. Subsequent binding analysis of HOX11-3'UTR and HOX113'UTR $\Delta$ with increased concentration of HuR was made and the results were quantitatively assayed. Fig. 5 shows the difference of binding ability between HOX11-3'UTR with HuR and HOX11-3'UTRA with HuR. When the HuR concentration is $800 \mathrm{nM}$, the percentage RNA bound value of HOX11-3'UTR $\Delta$ is less than $15 \%$. Compared with the strong 


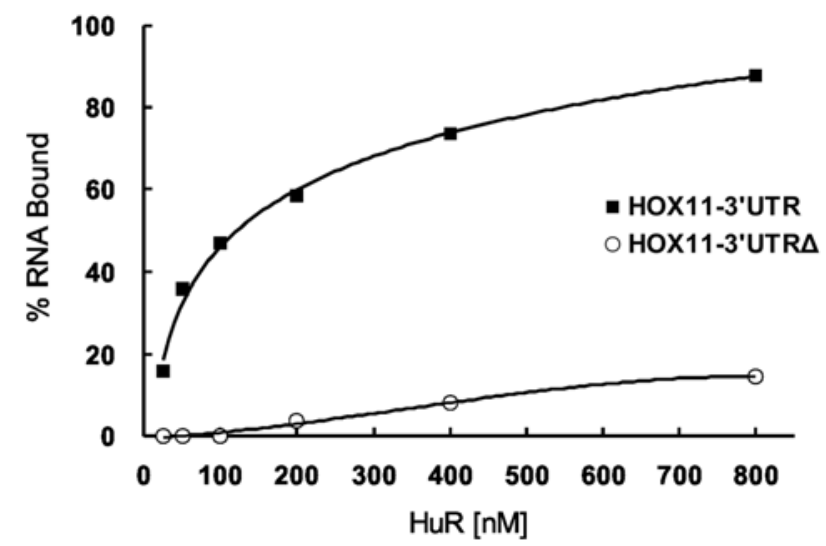

Fig. 5. Comparison of the binding ability between HuR with HOX11-3'UTR and HuR with HOX11-3'UTR $\triangle$. EMSA of HuR protein with biotin-labeled HOX11-3'UTR or HOX11-3'UTRA was performed as described above. The results were quantitatively analyzed and plotted.

binding affinity of HOX11-3'UTR with HuR, the binding ability of HOX11-3'UTRA with HuR is neglectable. It provides strong evidence that the AU-rich region contributes greatly to the specific binding of $H O X 11$ mRNA 3'UTR with HuR protein.

\section{Discussion}

In this paper, our results demonstrated that the 3'UTR of HOX11 mRNA could be bound by HuR protein specifically and this binding could be specifically competed by AUFL. An AU-rich segment contributes greatly to this specific binding. The results suggest that 3'UTR of HOXI1 mRNA contains a cis-element that is most likely to play a role in the posttranscriptional regulation.

Previous reports mainly focused on $\mathrm{HOX} 11$ regulation at the transcriptional level. Both positive and negative elements in the promoter of $H O X 115^{\prime}$ non-coding region on the chromosome were found (Brake et al., 1998; Brake et al., 2002). A recent research found that expression of HOXI1 in T-ALL is associated with extensive demethylation of the proximal HOX11 promoter (Watt et al., 2000). It was reported that $H O X 11$ expression is dependent on protein synthesis and its up-regulation in T cells requires a tyrosine phosphorylation signal (Zhang et al., 1995).

In addition to the transcriptional regulatory elements, posttranscriptional regulatory elements are emerging as an important control element for gene expression in eukaryotes (Sachs 1993; Ross 1995). ARE is the best-studied cis-element in mammalian mRNA, which will influence the mRNAs stability by acting with RNA-binding proteins. Many growth factors and cytokines integral to tumor proliferation and angiogenesis have ARE within the 3'UTR that govern transcript half-life (Chen and Shyu, 1995). HOX11 mRNA
3'UTR contains a cis-element suggests there may exist a posttranscriptional regulation of the $H O X 11$ gene expression. This post-transcriptional regulation may be a complementary mechanism for the development of spleen during embryogenesis or the deregulation of $H O X 11$ in T-ALL, which is believed to be a key event in the development of leukemia ( $\mathrm{Lu}$ et al., 1992).

The molecular mechanism of post transcriptional regulation depends on the interaction between the cis-element on the HOX11 mRNA 3'UTR and its binding factors. Many factors have been found to selectively bind the AU- and U-rich sequence, while only two of these proteins, $\operatorname{HuR}$ (Ma et al., 1996; Fan and Steitz, 1998; Brennan and Steitz 2001) and hnRNP D (AUF1) (Zhang, W. et al., 1993; Sarkar et al., 2003; Brewer et al., 2003) have been demonstrated to alter the stability of ARE-containing mRNA in vivo. HuR is a ubiquitously expressed member of the embryonic lethal abnormal vision (ELAV) family of human RNA-binding proteins (Ma et al., 1996). Overexpression of HuR protein stabilizes ARE-containing mRNA in vivo, which influences the final protein expression of the ARE-containing mRNA (Fan and Steitz, 1998; Peng et al., 1998; Levy et al., 1998). Recent evidences have suggested that HuR-ARE specific interaction plays a role in carcinogenesis by stabilizing AREcontaining mRNAs of growth factors and cytokines integral to tumor proliferation and angiogenesis. For example, HuR was found over-expressed in tumors of central nervous system, strong HuR expression was limited to high grade malignancies, and it was also found HuR could bind specifically to the ARE of angiogenic factors including vascular endothelial growth factor (VEGF), cyclooxygenase-2 (COX-2) and interleukin-8 (IL-8) (Nabors et al., 2001). In human colon cancer, the binding of HuR with the 3'UTR of cyclin-dependent kinase inhibitor p21 and carcinogenesis related gene VEGF, COX-2, IL-8 improves mRNA's stability (Wang, 2000; Dixon et al., 2001). In our result, we found HuR binding specifically to the HOX11 mRNA 3'UTR, which suggests that HuR may bind and stabilize HOX11 mRNA. It may be involved in the deregulation of $H O X 11$ in T-ALL.

Although the in vivo function of HuR in the regulation of $H O X 11$ expression need to be clarified, HuR protein showed its effectiveness in study of $\mathrm{HOX} 11$-HuR interaction in vitro. The different migration rate of RNA-protein complex in the EMSA reflects the heterogeneity of the complex and may be caused by the aggregation of RNA-protein complex.

Further study of the functional aspects of HuR-HOX11 3'UTR interactions may provide information on the regulation of the gene in vivo. The HOX11 ARE or its mutations could be inserted downstream of reporter gene for the transfection of different cell types to examine the influence and effectiveness on the post-transcriptional regulation of reporter gene in vivo. This study would help to understand the critical structure features and the function of the ARE that may be involved in the $H O X 11$ gene regulation at the post-transcriptional level. A further scanning of the HuR expression level and experimental 
manipulation in T-ALL would also help to explore the possible role of HuR in the HOXI1 deregulation in T-ALL.

Acknowledgments This work was supported by grant from Shanghai-Anson Research Foundation and grant from Chinese Academy of Science.

\section{References}

Bradford, M. M. (1976) A rapid and sensitive method for the quantitation of microgram quantities of protein utilizing the principle of protein-dye binding. Anal. Biochem. 72, 248-254.

Brake, R. L., Kees, U. R. and Watt, P. M. (1998) Multiple negative elements contribute to repression of the HOX11 protooncogene. Oncogene 17, 1787-1795.

Brake, R. L., Kees, U. R. and Watt, P. M. (2002) A complex containing PBX2 contributes to activation of the protooncogene HOX11. Biochem. Biophys. Res. Commun. 294, 2334.

Brennan, C. M. and Steitz, J. A. (2001) HuR and mRNA stability. Cell Mol. Life Sci. 58, 266-277.

Brewer, G., Saccani, S., Sarkar, S., Lewis, A. and Pestka, S. (2003) Increased interleukin-10 mRNA stability in melanoma cells is associated with decreased levels of A + U-rich element binding factor AUF1. J. Interferon Cytokine Res. 23, 553-564.

Chen, C. Y. and Shyu, A. B. (1995) AU-rich elements: characterization and importance in mRNA degradation. Trends Biochem. Sci. 20, 465-470.

Chung, S., Jiang, L., Cheng, S. and Furneaux, H. (1996) Purification and properties of $\mathrm{HuD}$, a neuronal RNA-binding protein. J. Biol. Chem. 271, 11518-11524.

Dear, T. N., Colledge, W. H., Carlton, M. B., Lavenir, I., Larson, T., Smith, A. J., Warren, A. J., Evans, M. J., Sofroniew, M. V. and Rabbitts, T. H. (1995) The Hoxll gene is essential for cell survival during spleen development. Development 121, 29092915.

Dear, T. N. and Rabbitts, T. H. (1994) A Drosophila melanogaster homologue of the T-cell oncogene HOX11 localises to a cluster of homeobox genes. Gene 141, 225-229.

Dear, T. N., Sanchez-Garcia, I. and Rabbitts, T. H. (1993) The HOX11 gene encodes a DNA-binding nuclear transcription factor belonging to a distinct family of homeobox genes. Proc. Natl. Acad. Sci. USA 90, 4431-4435.

Dixon, D. A., Tolley, N. D., King, P. H., Nabors, L. B., McIntyre, T. M., Zimmerman, G. A. and Prescott, S. M. (2001) Altered expression of the mRNA stability factor HuR promotes cyclooxygenase- 2 expression in colon cancer cells. J. Clin. Invest. 108, 1657-1665.

Fan, X. C. and Steitz, J. A. (1998) Overexpression of HuR, a nuclear-cytoplasmic shuttling protein, increases the in vivo stability of ARE-containing mRNAs. EMBO J. 17, 3448-3460.

Kanzler, B. and Dear, T. N. (2001) Hox11 acts cell autonomously in spleen development and its absence results in altered cell fate of mesenchymal spleen precursors. Dev. Biol. 234, 231243.

Kees, U. R., Heerema, N. A., Kumar, R., Watt, P. M., Baker, D. L., La, M. K., Uckun, F. M. and Sather, H. N. (2003) Expression of HOXI1 in childhood T-lineage acute lymphoblastic leukaemia can occur in the absence of cytogenetic aberration at 10q24: a study from the Children's Cancer Group (CCG). Leukemia 17, 887-893.

Kennedy, M. A., Gonzalez-Sarmiento, R., Kees, U. R., Lampert, F., Dear, N., Boehm, T. and Rabbitts, T. H. (1991) HOX11, a homeobox-containing T-cell oncogene on human chromosome 10q24. Proc. Natl. Acad. Sci. USA 88, 8900-8904.

Levy, A. P., Levy, N. S. and Goldberg, M. A. (1996) Posttranscriptional regulation of vascular endothelial growth factor by hypoxia. J. Biol. Chem. 271, 2746-2753.

Levy, N. S., Chung, S., Furneaux, H. and Levy, A. P. (1998) Hypoxic stabilization of vascular endothelial growth factor mRNA by the RNA-binding protein HuR. J. Biol. Chem. 273, 6417-6423.

Li, Y., Jiang, Z. Z., Chen, H. X. and Ma, W. J. (2004) A modified quantitative EMSA and its application in the study of RNAprotein interactions. J. Biochem. Biophys. Methods 60, 85-96.

Lichty, B. D., Ackland-Snow, J., Noble, L., Kamel-Reid, S. and Dube, I. D. (1995) Dysregulation of HOX11 by chromosome translocations in T-cell acute lymphoblastic leukemia: a paradigm for homeobox gene involvement in human cancer. Leuk. Lymphoma. 16, 209-215.

Lu, M., Zhang, N. and Ho, A. D. (1992) Genomic organization of the putative human homeobox proto-oncogene HOX-11 (TCL3 ) and its endogenous expression in T cells. Oncogene 7, 1325-1330.

Ma, W. J., Cheng, S., Campbell, C., Wright, A. and Furneaux, H. (1996) Cloning and characterization of HuR, a ubiquitously expressed Elav-like protein. J. Biol. Chem. 271, 8144-8151.

Nabors, L. B., Gillespie, G. Y., Harkins, L. and King, P. H. (2001) HuR, a RNA stability factor, is expressed in malignant brain tumors and binds to adenine- and uridine-rich elements within the $3^{\prime}$ untranslated regions of cytokine and angiogenic factor mRNAs. Cancer Res. 61, 2154-2161.

Patterson, K. D. and Krieg, P. A. (1999) Hox11-family genes XHox11 and XHox11L2 in xenopus: XHox11L2 expression is restricted to a subset of the primary sensory neurons. Dev. Dyn. 214, 34-43.

Peng, S. S., Chen, C. Y., Xu, N. and Shyu, A. B. (1998) RNA stabilization by the AU-rich element binding protein, HuR, an ELAV protein. EMBO J. 17, 3461-3470.

Roberts, C. W., Shutter, J. R. and Korsmeyer, S. J. (1994) Hox11 controls the genesis of the spleen. Nature 368, 747-749.

Ross, J. (1995) mRNA stability in mammalian cells. Microbiol. Rev. 59, 423-450.

Sachs, A. B. (1993) Messenger RNA degradation in eukaryotes. Cell 74, 413-421.

Sambrook, J., Fritsch, E. F. and Maniatis, T. (1992) Molecular Cloning. A Laboratory Manual, 2nd ed., Cold Spring Harbor, New York, USA.

Sarkar, B., Xi, Q., He, C. and Schneider, R. J. (2003) Selective degradation of AU-rich mRNAs promoted by the p37 AUF1 protein isoform. Mol. Cell Biol. 23, 6685-6693.

Shaw, G. and Kamen, R. (1986) A conserved AU sequence from the $3^{\prime}$ untranslated region of GM-CSF mRNA mediates selective mRNA degradation. Cell 46, 659-667.

Shyu, A. B., Belasco, J. G. and Greenberg, M. E. (1991) Two distinct destabilizing elements in the c-fos message trigger deadenylation as a first step in rapid mRNA decay. Genes Dev. 5, 221-231 
Wang, W., Furneaux, H., Cheng, H., Caldwell, M. C., Hutter, D., Liu, Y., Holbrook, N. and Gorospe, M. (2000) HuR regulates p21 mRNA stabilization by UV light. Mol. Cell. Biol. 20, 760769.

Watt, P. M., Kumar, R. and Kees, U. R. (2000) Promoter demethylation accompanies reactivation of the HOX11 protooncogene in leukemia. Genes Chromosomes Cancer 29, 371377.

Zhang, N., Gong, Z. Z., Minden, M. and Lu, M. (1993) The HOX-11 (TCL-3) homeobox proto-oncogene encodes a nuclear protein that undergoes cell cycle-dependent regulation. Oncogene 8, 3265-3270.
Zhang, N., Shen, W., Ho, A. D. and Lu, M. (1996) Three distinct domains in the HOX-11 homeobox oncoprotein are required for optimal transactivation. Oncogene 13, 1781-1787.

Zhang, N., Shen, W. F., Ho, A. D. and Lu, M. (1995) Tyrosine phosphorylation is required for up-regulation of the $\mathrm{HOX}-11$ (TCL-3) homeobox proto-oncogene in T cells. Cancer Res. 55, 1117-1121.

Zhang, W., Wagner, B. J., Ehrenman, K., Schaefer, A. W., DeMaria, C. T., Crater, D., DeHaven, K., Long, L. and Brewer, G. (1993) Purification, characterization, and cDNA cloning of an AU-rich element RNA-binding protein, AUF1. Mol. Cell Biol. 13, 7652-7665. 